\title{
Constructing an Electronic Circuitry for Label-free Hall Biosensors
}

\author{
D. IZCI, J. HEDLEY
}

\begin{abstract}
Magnetic field has a huge potential of providing non-destructive and highly efficient detection platforms in biosensing field. It provides a low intrinsic background in biological systems since those systems have no comparable biological signal. Hall devices are currently dominating the market of the magnetic sensors due to several advantages such as allowing miniaturization, being compatible with electronics integration, cheaper fabrication and room temperature operation with high linearity. It is important to construct a suitable frontend circuitry in order to be able to achieve a portable and labelfree working Hall biosensor. This paper presents the construction of an electronic circuitry that is required for actuating Hall based biosensors and obtaining the output since there are undesired effects which are causing less sensitive or less accurate results. The paper takes a closer look at those effects and presents the solution to the problem by introducing a developed circuitry on a designed printed circuit board along with the promising results it has achieved.
\end{abstract}

Index Terms - Hall effect, Hall biosensors, Offset removal, Spinning current circuitry.

\section{INTRODUCTION}

$\mathrm{H}$ ALL EFFECT is one of the fundamental techniques used for characterizing electrical transport properties in metals and semiconductors. The basic idea of Hall effect is to drive current through a thin layer of metal sheet or semiconductor and apply a magnetic field perpendicular to the driven current. This exerts a force which is perpendicular to both driven current and applied magnetic field. The exerted force is also known as Lorentz Force [1] and is illustrated in Fig. 1. Transport properties of a material such as carrier mobility, carrier concentration and carrier type can be determined by measuring Hall voltage.

DAVUT IZCI, is with Department of Computer Engineering Batman University, Batman, Turkey, (e-mail: davut.izci@batman.edu.tr).

(iD) https://orcid.org/0000-0001-8359-0875

JOHN HEDLEY, is with School of Engineering Newcastle University, Newcastle upon Tyne, UK, (e-mail: john.hedley@ncl.ac.uk).

(iD) https://orcid.org/0000-0002-2893-8776

Manuscript received June 22, 2019; accepted September 01, 2019. DOI: $\underline{10.17694 / \text { bajece. } 633908}$

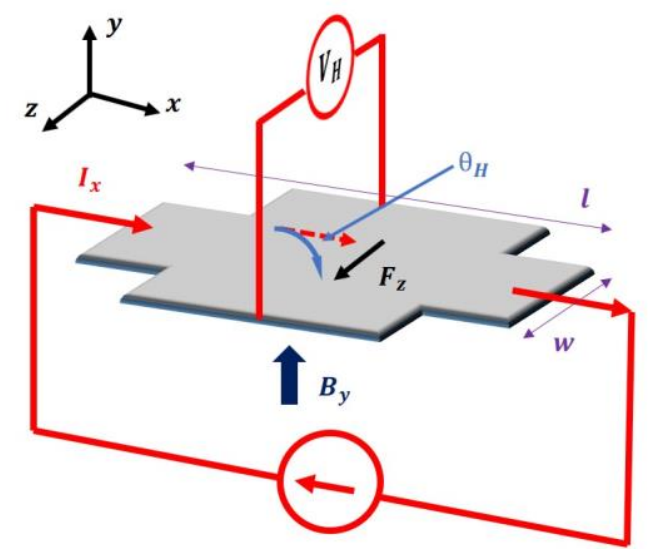

Fig. 1. A representation of Hall sensing principle.

The voltage exerted from the system is proportional with carrier mobility and inversely proportional with carrier concentration and thickness of the material as stated in Eq.(1). In Eq.(1), the term $\mathrm{V}_{\mathrm{H}}$ represents the Hall voltage, $n$ denotes the density of the charge carriers (carrier concentration) and it is given in $\mathrm{cm}^{-2}$ unit, $\mu$ is for carrier mobility with the unit of $\mathrm{cm}^{2} \mathrm{~V}^{-1} \mathrm{~s}^{-1}$ and e stands for electron charge $\left(\sim 1.60 \times 10^{-19}\right.$ coulombs). Additionally, $\mathrm{t}$ denotes the thickness of the material in metres, $\mathrm{I}_{\mathrm{x}}$ represents the applied current that flows through the material sheet, $E_{x}$ is the applied electric field and $\mathrm{B}_{\mathrm{y}}$ is the term that represents the perpendicular magnetic field. $\mathrm{G}$ represents geometrical factor [2] which depends on carrier mobility and length to width ratio $(l / w)$.

$$
V_{H}=G \frac{1}{n e} \frac{B_{y} I_{x}}{t}
$$

The Hall effect phenomenon has found important roles for many practical applications in last decades by utilizing Hall sensors. Those sensors are magnetic type sensors that are using the perpendicular magnetic field component to produce information and have been investigated for more than a hundred years and used for various purposes since then [3][5]. They are used widely in many applications as low-cost sensors. The applicability of Hall principle has also been investigated quite recently for biomedical purposes [6] and a considerable number of reports have been devoted to employability of Hall principle for biosensing applications [7]-[12]. Hall based biosensors have been shown to be successful with the aid of magnetic particles [13]-[16]. In this type of detection, magnetic beads are used to measure magnetic susceptibility [14]. Basically, a combination of 
alternating and non-alternating magnetic fields is applied inplane and perpendicular to the sensor surface, respectively (see Fig. 2). The detection is achieved via observing an output signal which has the same frequency of the in-plane excitation signal.

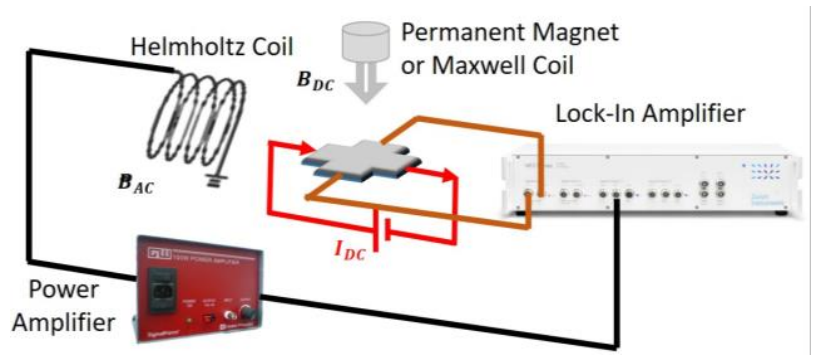

Fig. 2. Schematic view of the ac susceptibility measurements.

However, there are some restrictions that limit the sensitivity of the Hall sensor if magnetic beads are used. It is crucial to consider the separation between surface of the sensor and magnetic bead as magnetic field from the beads decreases proportional to $\sim 1 / \mathrm{d}^{3}$ where $\mathrm{d}$ represents the separation distance [17]. This parameter must be treated carefully for an optimized signal to noise ratio. Additionally, magnetic moment of a magnetic bead is another important parameter that must be considered since those parameters are affecting the magnetic field provided by magnetic beads. Moreover, it is quite hard to observe the output in real-time for a longer period in this type of detection scheme since it requires more power due to complexity of electronics. Running a coil for longer would also affect the produced magnetic output because of thermal heat being produced. Because of the complexity it requires in terms of electronics setup, as shown in Fig. 2, creating such a portable system is not feasible.

It is important to scale down the system due to necessity of real-time monitoring and point-of-care system requirements. Therefore, a label-free approach seems more attractive. However, an electronic front-end would be required to make a label-free system achievable and cost-effective along with good performance parameters. This paper investigates the steps and presents a solution to make the sensors portable with significantly improved performances.

\section{DETECTION MECHANISM FOR LABEL-FREE APPROACH}

A Hall effect sensor can be thought of as a combination of three major parts; the transducer itself, power supply to bias the transducer and data acquisition stage for acquiring relevant output. However, those parts require to be used in conjunction with specially designed electronics to perform properly and to present the data to end user. Adopting specially designed electronics significantly improves the system capability by maintaining the performance and providing application specific data, thus, leaving no concern to the end user.

\section{A. Biasing Source}

A Hall transducer can, in principle, be used simply by adding a stable power supply to it and biasing can be achieved either with a voltage or a current source. A simple setup for biasing a Hall device is demonstrated in Fig. 3. In case of a voltage source, the temperature coefficient of the transducer sensitivity will be as high as $0.3 \%{ }^{\circ} \mathrm{C}$ [3], meaning that the output will vary significantly with respect to temperature change. Therefore, some additional front-end circuitry is required to limit the current that will be sourced from the voltage supply and provide more stable output in case of voltage mode of operation [3], [18]. Limiting the current is particularly important to reduce the power consumption, hence, preventing device from thermal heat effect. To obtain a stable output in case of temperature changes, the voltage source or the gain of the amplifier can be made temperature dependent [1] to maintain a stable output, thus, making the system less sensitive to variations of temperature. In terms of implementation, a buffer must be placed in between voltage reference and the Hall transducer for low current transducers where an additional transistor can be added in between the buffer and the device for the transducers requiring high currents.

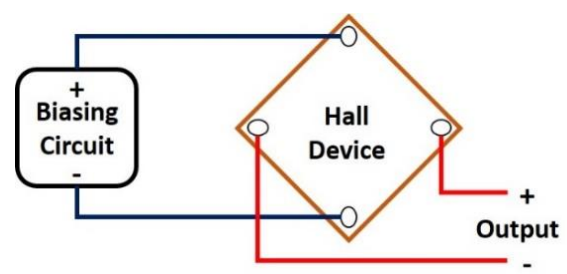

Fig. 3. An illustration of biasing a Hall plate.

Another way of reducing the output variations with respect to temperature changes is to bias the transducer via a current source. Current mode of operation provides a reduction as low as $0.05 \%{ }^{\circ} \mathrm{C}$ in temperature coefficient [3]. As a result, current source biasing is preferable in most cases as it provides more stable working conditions due to significant reduction in temperature coefficient [19]. In terms of current mode of operation, a power supply can simply be used with a combination of resistors for biasing devices. A constant current biasing circuit can be achieved by placing simply a resistor in between voltage reference and the transducer. It can also be obtained via using a transistor by connecting it to the transducer as shown in Fig. 4.

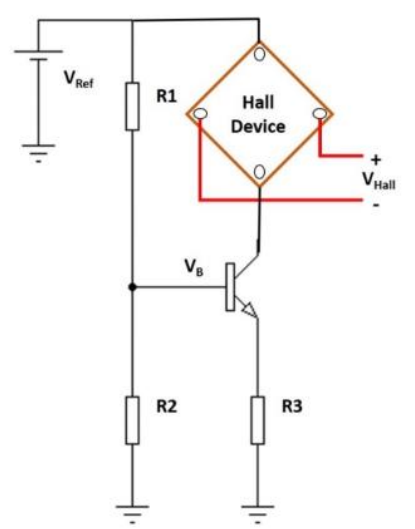

Fig. 4. Biasing Hall transducer via a constant current source using a transistor. 


\section{B. Amplification}

In general, Hall voltages are quite small and therefore require amplification. Therefore, it is necessary to implement an amplification process since the output of such systems are in millivolt or microvolt ranges. Depending on the structure of the devices, the Hall voltage might be even smaller. The smaller ranges make it difficult to measure since it is below the detectable range of most of the acquisition systems. In the amplification stage, an instrumentation amplifier is desired since Hall devices have differential outputs as seen in Fig. 5.

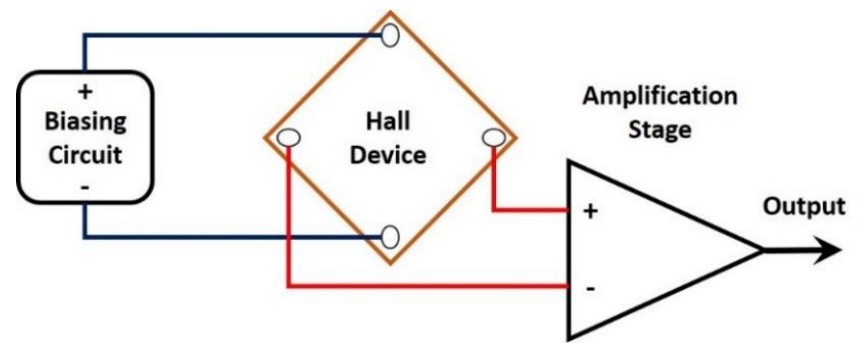

Fig. 5. Implementation of an amplification stage

\section{Offset Removal}

The offset voltage is an undesired effect which occurs due to contact misalignments, poor surface roughness or nonhomogeneous device structure [20] and introduces a non-zero voltage value for the cases of zero magnetic field and keeps increasing with increased current under constant magnetic field. In most cases, this voltage could be even higher than the actual Hall voltage. Fig. 6 represents the parameters that causes the offset voltage. The amplification stage increases the output and help better understanding of the voltage change; however, it still has the limitations in terms of the ability of accurate measurement since the output is amplified together with offset voltage. That means the amplification process also causes the amplification of the offset voltage, as well.

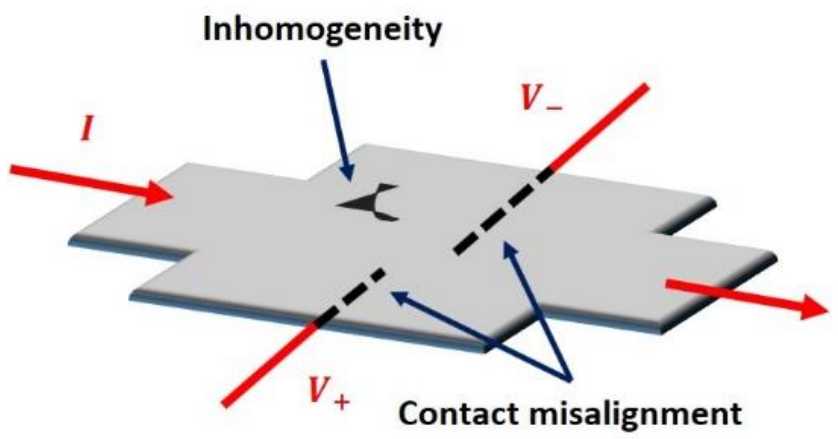

Fig. 6. The parameters that causes offset voltage.

A potentiometer can be used as a tool for eliminating offset voltage by being connected to the output of the amplification circuit as shown in Fig. 7 and could be arranged to provide zero offset. However, this will work for a specific biasing current and will require an adjustment if the amount of biasing current changes. Additionally, thermal effects will be dominant since a potentiometer is in use.

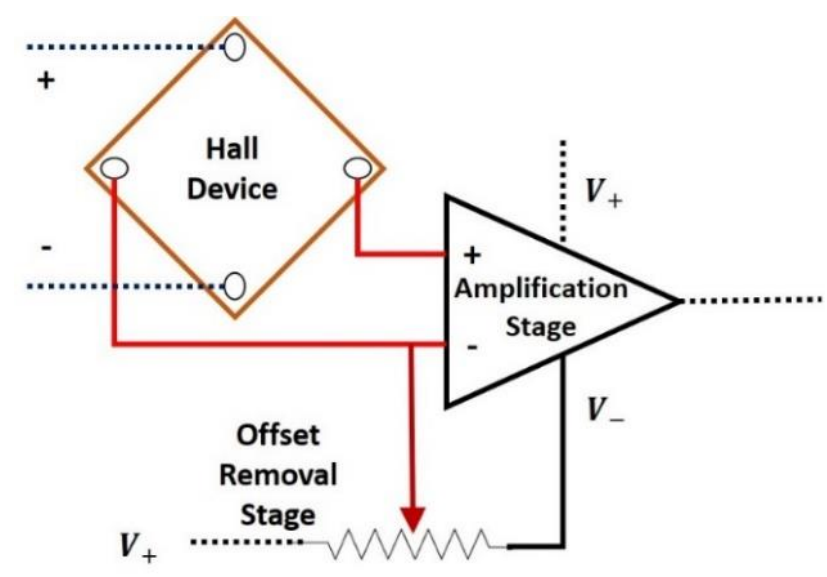

Fig. 7. A basic offset removal stage for Hall devices using a potentiometer.

\section{Data Acquisition}

Data acquisition can be performed after the amplification and offset removal stages. The amplification stage introduces another issue which might cause a deteriorating effect on actual signal of interest since it leads to a similar amount of amplification of noise signals. Therefore, the output of the amplifier must be filtered with a low pass filter for removal of undesired frequency components. Fig. 8 shows the implementation of a filtering stage. A filter having a specified cut-off frequency can simply be integrated to the circuit via passive components such as capacitors and resistors. Meanwhile, it can also be filtered using a software after acquisition.

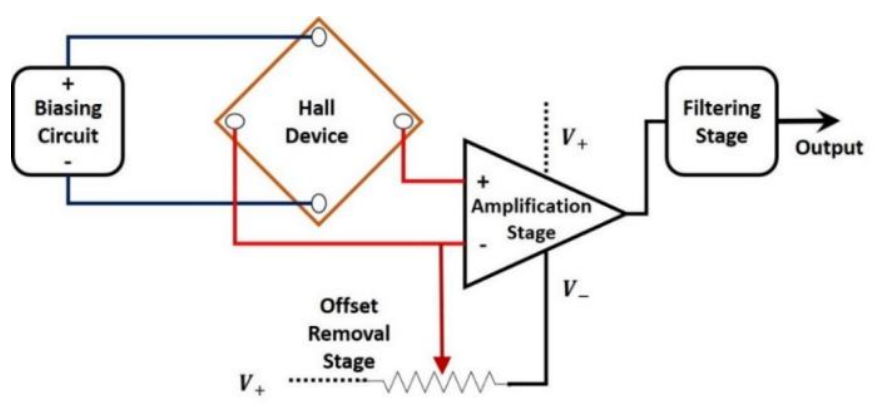

Fig. 8. Basic biasing, amplifying, offset removal and filtering stages.

\section{DEVELOPMENT OF AN INTEGRATED CIRCUITRY}

The approaches presented in above sections will not be able to remove the offset dynamically since any change in the biasing current leads to a change in output regardless of magnetic field. More importantly, the output is prone to thermal effects in such a design since it becomes very sensitive to temperature changes. To remove the offset voltage, a technique called 'current spinning' was employed [21] which can remove the offset voltage dynamically. Fig. 9 is a representation of a cross shaped Hall device which will be used to explain the spinning method between contacts. Basically, the idea can be explained by a simple approximation of a Hall device structure by using four resistors connected to each other in a Wheatstone bridge structure. 


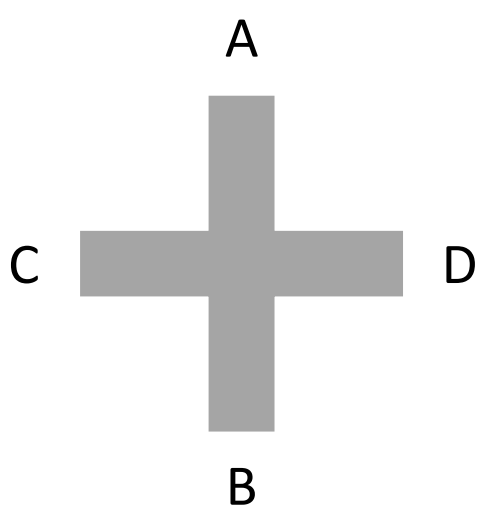

Fig. 9. A representation of cross shape Hall device.

In this case, an offset voltage can easily be introduced by using three resistors with the same values and one with a different value. Once the current is applied from one connection to the other non-neighboring connection a voltage will be measured between other two non-neighboring connection points. If this setup is rotated by $180^{\circ}$, the measured voltage will change in sign but remain as the same value. In case of a Hall device, the Hall voltage would not change since it depends on the exerted force due to applied current and magnetic field. Once the outputs for $0^{\circ}$ and $180^{\circ}$ are obtained, the Hall voltage can easily be calculated by doing a simple mathematical operation on them. This method is summarized in TABLE 1 by considering the current rotation between all contacts.

TABLE 1. BIASING AND MEASUREMENT CONFIGURATIONS FOR A CROSS SHAPE HALL DEVICE

\begin{tabular}{ccc}
\hline Current & Measure & Obtained Output \\
\hline From $A$ to $B$ & Between $C$ and $D$ & $V_{H}+V_{\text {offset1 }}$ \\
\hline From $C$ to $D$ & Between $B$ and $A$ & $V_{H}+V_{\text {offset2 }}$ \\
\hline From $B$ to $A$ & Between $D$ and $C$ & $V_{H}-V_{\text {offset1 }}$ \\
\hline From $D$ to $C$ & Between $A$ and $B$ & $V_{H}-V_{\text {offset2 }}$ \\
\hline
\end{tabular}

To address the issue, a bespoke circuitry was designed and constructed on a PCB board, by employing a 'current spin model' [20], and integrated with the graphene Hall effect sensors for biasing and processing the output. The circuitry was used to correct the output by eliminating non-desired offset voltages and reducing noise levels thus providing an improvement on the sensitivity of the overall system. The schematic in Fig. 10 demonstrates the steps that were implemented for driving devices and reading the outputs. Fundamentally, the current is driven from one of the contacts to a non-neighboring contact and simultaneously the produced output voltage is measured across the remaining two contacts (e.g. current flows from contact $\mathrm{A}$ to $\mathrm{B}$ and the voltage difference between $\mathrm{C}$ and $\mathrm{D}$ is measured (Fig. 9 and TABLE 1). In such a case, the produced output includes both Hall voltage $\left(V_{H}\right)$ generated by applied perpendicular magnetic field $\left(B_{y}\right)$ and offset voltage $\left(V_{\text {off }}\right)$. To be able to remove the offset voltage, the current flow is rotated $180^{\circ}$ and passes through two new non-neighboring contacts whilst the voltage across the remaining contacts is measured. In this case, the measured output includes Hall voltage subtracted by offset voltage. For a more robust elimination, this procedure continues for one complete cycle meaning that the current is driven between all contacts for a complete cycle following $90^{\circ}$ rotation steps. Averaging the obtained outputs eliminates the offset and reveals the Hall voltage.

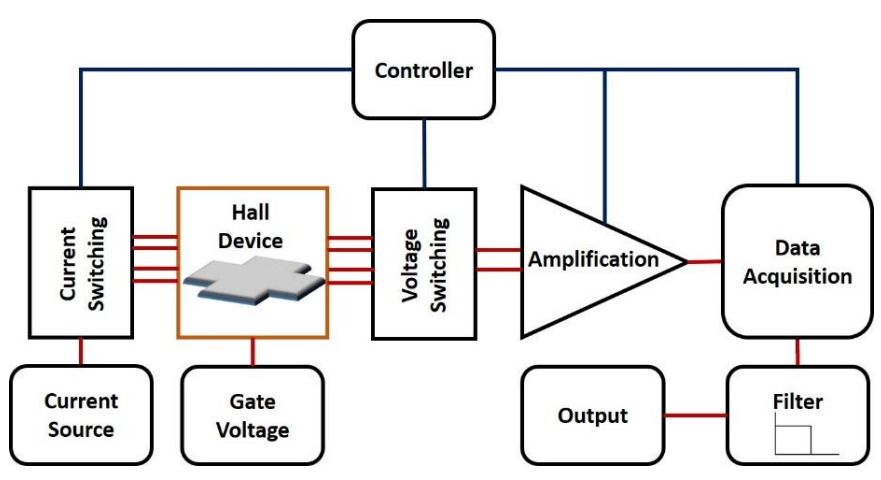

Fig. 10. Schematic of developed driving and processing circuitry.

The constructed PCB (Fig. 11) has an on-board constant current source, which can easily be adjusted from $1 \mu \mathrm{A}$ to 10 $\mathrm{mA}$ and can bias the sensor in floating or grounded mode of operation. Due to the controllable sensitivity of materials such as graphene, an optional input for gate voltage was also included. Current and voltage switching mechanisms were connected to the current source and the amplifier along with the connections to device contacts. A microcontroller simultaneously manipulates the current rotation and output reading for various frequencies up to $1 \mathrm{MHz}$. Amplification was performed via a high-performance instrumentation amplifier (LMP8358). The sensors can be integrated by being mounted on the tip of the board as shown in the figure. A rare earth magnet such as neodymium with relevant dimensions can easily be fitted to the tip of the developed portable system given in Fig. 11.

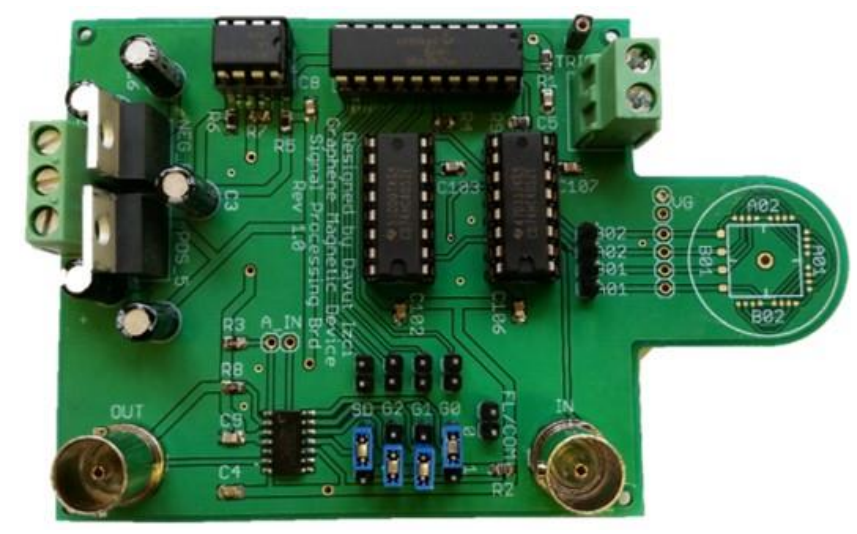

Fig. 11. Constructed system board PCB.

\section{RESULTS AND DISCUSSION}

The Hall voltage is not affected from the misalignment of the contacts along the length of the device. Because the electrons would feel the same amount of resistance to their 
flow and a voltage value due to the exerted force would occur at the location where the transverse contacts are placed. A typical output obtained from a Hall device without using the developed front-end circuitry is shown in Fig. 12 as an example.

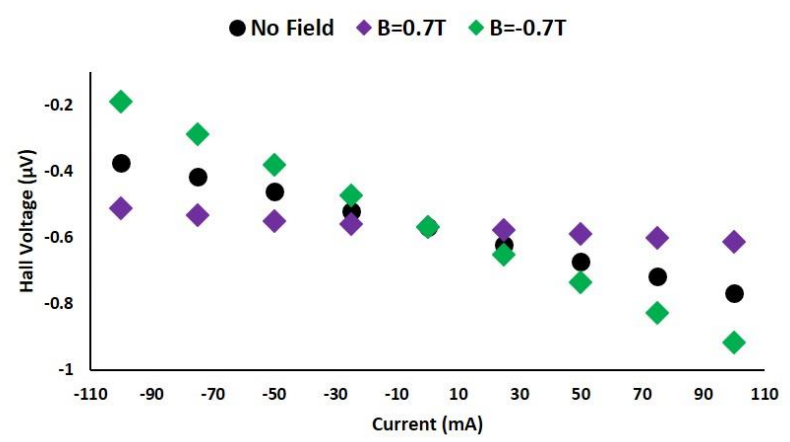

Fig. 12. A typical output obtained from a Hall device without correction.

The zero-magnetic field line should have been on zerohorizontal line instead of being a negative value with a voltage gradient. This was because of the offset voltage effect as explained in previous sections. However, if the no-field line is taken as a reference, the response of the device is highly linear for both positive and negative magnetic field strengths since a voltage gradient is observed for positive and negative values of current and magnetic field. A typical processed output of the device for one cycle (using the developed front-end circuitry) is given in Fig. 13.

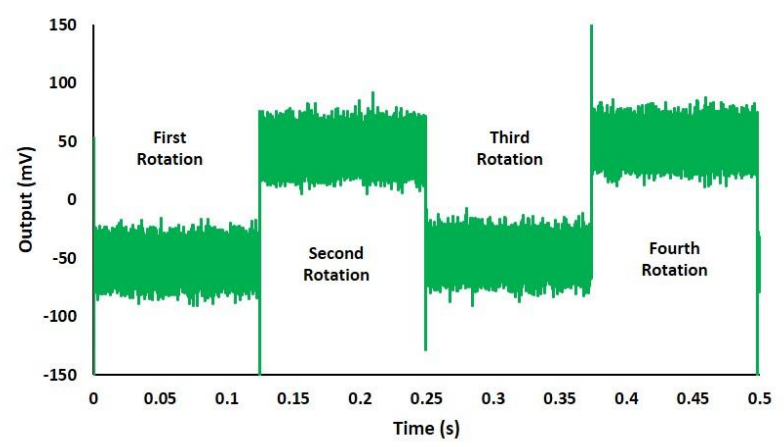

Fig. 13. A Typical output obtained after one cycle.

Each region indicated in the figure corresponds to the specific cases related to current rotation sequences, e.g., first region is the obtained output for the first case given in TABLE 1 and the second region is the obtained output for the second case given in TABLE 1, and this sequence keeps going on. The response of the device shown in Fig. 12 was corrected using the developed circuitry. Fig. 14 presents the corrected version of the results. As seen clearly, the voltage gradient is removed, and the voltage of interest is presented.

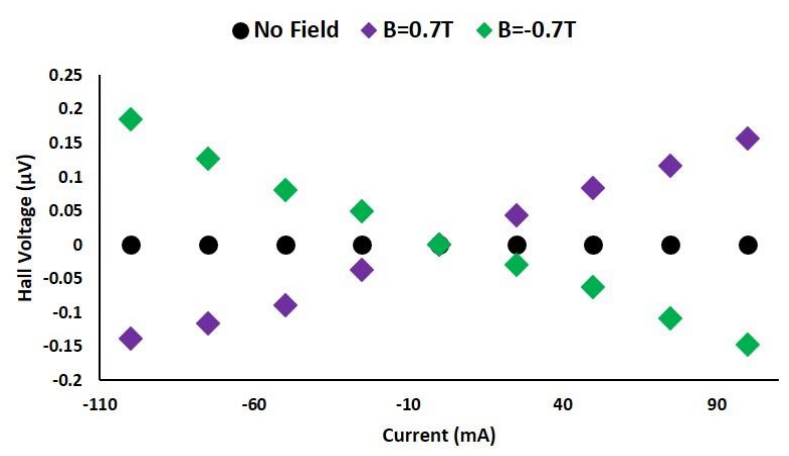

Fig. 14. Corrected Hall output after removing offset voltage.

The developed driving and processing board was tested for its dynamic offset removal ability. The results obtained from the output of the devices with and without circuit implementation was initially assessed for no magnetic field with a variable current source Fig. 15. Direct driving of the Hall sensor under no magnetic field has shown significant reduction in offset voltage. The data shown with purple provides the reduction ratio in percentage.

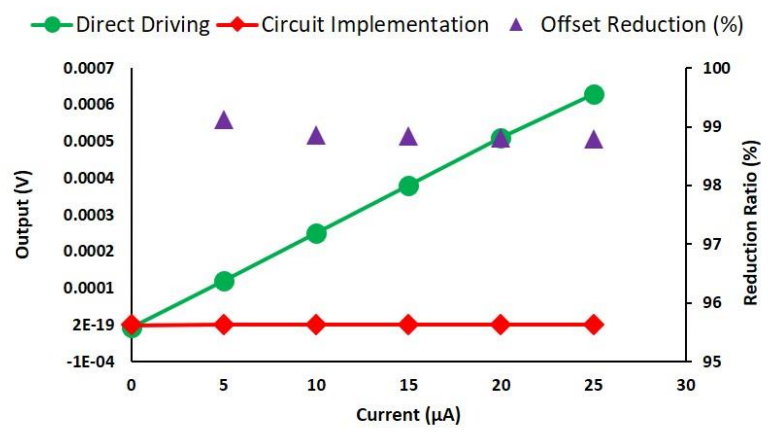

Fig. 15. Demonstration of offset removal.

The circuitry was also tested for constant current flow with variable magnetic field as shown in Fig. 16. The implementation of the circuitry helped reducing the offset voltage by $99 \%$. The negligible residual offset shown in Fig. 17 corresponded to an offset equivalent magnetic field value of $100 \mathrm{nT}$ thus providing a significant improvement in terms of the system accuracy.

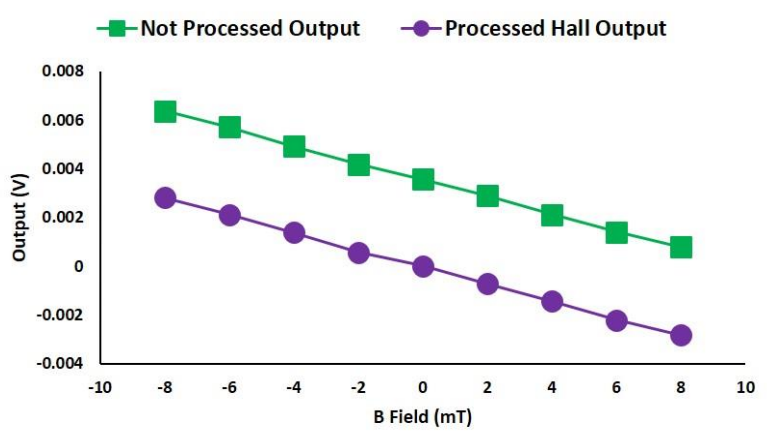

Fig. 16. Processed and not processed outputs for variable magnetic fields. 


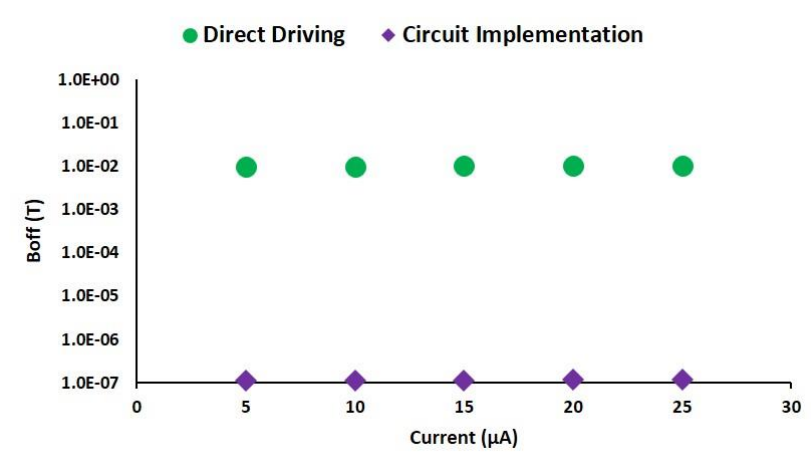

Fig. 17. The comparison of residual magnetic offset values.

Also, fast Fourier transform measurements with and without biasing and driving circuitry showed that the noise level is reduced considerably by employing the developed circuitry. The power spectral density figures showed that the higher frequency operation leads to lower noise effect, thus, providing devices with better sensitivities. Fig. 18 shows power spectral density measurements with respect to frequency of rotation.

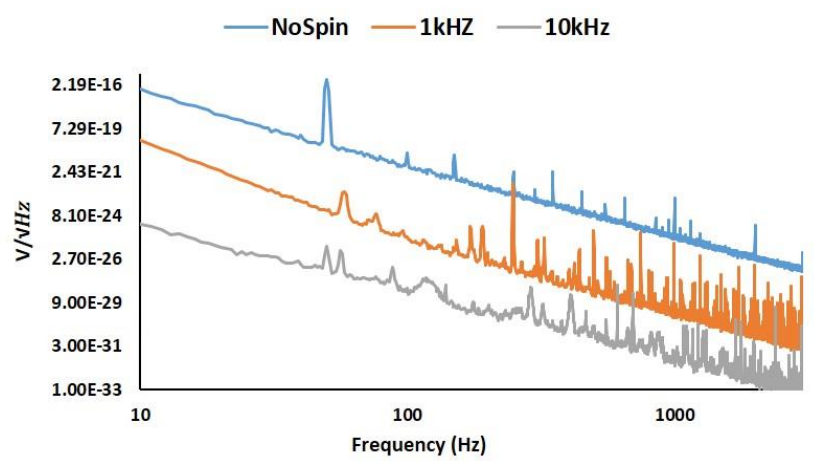

Fig. 18. Power spectral density measurements.

\section{CONCLUSION}

In this work, an electronic front-end circuitry has been designed and interfaced with Hall biosensors in order to obtain a portable system. The system was proved to have merit for potential point-of-care systems as it does not require to use labels such as beads, thus, eliminates complex electronics setup. In addition, the figures showed that the system has the capacity of correcting undesired effects such as offset voltage and reducing noise, therefore, making the system available for detecting lower field strengths. Being placed on a small printed circuit provides a compact and functional structure which can pave the way for future wearable biosensing systems.

\section{REFERENCES}

[1] R. S. Popović, Hall effect devices, 2nd ed. Philadelphia : Institute of Physics Pub., 2004.

[2] G. Boero, M. Demierre, P. A. Besse, and R. S. Popovic, "Micro-Hall devices: Performance, technologies and applications," Sensors Actuators, A Phys., vol. 106, no. 1-3, pp. 314-320, 2003.
[3] E. Ramsden, Hall-Effect Sensors, 2nd ed. Elsevier, 2006.

[4] H. $\mathrm{Xu}$ et al., "Batch-fabricated high-performance graphene Hall elements," Sci. Rep., vol. 3, p. 1207, 2013.

[5] D. Izci, C. Dale, N. Keegan, and J. Hedley, "The Construction of a Graphene Hall Effect Magnetometer," IEEE Sens. J., vol. 18, no. 23, pp. 9534-9541, Dec. 2018.

[6] S. Johnstone, "Is there potential for use of the Hall effect in analytical science?," Analyst, vol. 133, no. 3, pp. 293-296, 2008.

[7] K. Skucha, P. Liu, M. Megens, J. Kim, and B. Boser, "A compact Hall-effect sensor array for the detection and imaging of single magnetic beads in biomedical assays," 16th Int. Solid-State Sensors, Actuators Microsystems Conf. TRANSDUCERS'11, pp. 18331836, 2011.

[8] T. Ishikawa, "Immunoassay on silicon chip," 2014 29th Symp. Microelectron. Technol. Devices Chip Aracaju, SBMicro 2014, 2014.

[9] P. Manandhar et al., "The detection of specific biomolecular interactions with micro-Hall magnetic sensors," Nanotechnology, vol. 20, no. 35, p. 355501 , 2009.

[10] A. Sandhu and H. Handa, "Practical hall sensors for biomedical instrumentation," IEEE Trans. Magn., vol. 41, no. 10, pp. 4123-4127, 2005.

[11] K. Togawa et al., "High sensitivity InSb hall effect biosensor platform for DNA detection and biomolecular recognition using functionalized magnetic nanobeads," Japanese J. Appl. Physics, Part 2 Lett., vol. 44, no. 46-49, pp. L1494-L1497, 2005.

[12] D. Issadore, H. J. Chung, J. Chung, G. Budin, R. Weissleder, and $H$. Lee, " $\mu$ Hall chip for sensitive detection of bacteria," Adv. Healthc. Mater., vol. 2, no. 9, pp. 1224-1228, 2013.

[13] D. Issadore et al., "Magnetic sensing technology for molecular analyses," Lab Chip, vol. 14, no. 14, pp. 2385-2397, 2014.

[14] T. Takamura, P. J. Ko, J. Sharma, R. Yukino, S. Ishizawa, and A. Sandhu, "Magnetic-particle-sensing based diagnostic protocols and applications," Sensors (Switzerland), vol. 15, no. 6, pp. 12983-12998, 2015.

[15] T. A. P. Rocha-Santos, "Sensors and biosensors based on magnetic nanoparticles," TrAC - Trends Anal. Chem., vol. 62, pp. 28-36, 2014.

[16] J. Wang, A. N. Kawde, A. Erdem, and M. Salazar, "Magnetic bead-based label-free electrochemical detection of DNA hybridization," Analyst, vol. 126, no. 11, pp. 2020-2024, 2001.

[17] A. Manzin and V. Nabaei, "Modelling of micro-Hall sensors for magnetization imaging," J. Appl. Phys., vol. 115, no. 17, 2014.

[18] M. A. Paun, J. M. Sallese, and M. Kayal, "Hall effect sensors design, integration and behavior analysis," $J$. Sens. Actuator Networks, vol. 2, no. 1, pp. 85-97, 2013.

[19] S. Sanfilippo, "Hall probes: Physics and application to 
magnetometry," in CAS 2009 - CERN Accelerator School: Magnets, Proceedings, 2010, pp. 423-462.

[20] R. Steiner, C. Maier, A. Hàberli, F. P. Steiner, and H. Baltes, "Offset reduction in Hall devices by continuous spinning current method," Sensors Actuators, A Phys., vol. 66, no. 1-3, pp. 167-172, 1998.

[21] X. Chen, Y. Xu, X. Xie, Y. Guo, and Y. Huang, "A novel Hall dynamic offset cancellation circuit based on four-phase spinning current technique," China Semicond. Technol. Int. Conf. 2015, CSTIC 2015, pp. $1-3,2015$.

\section{BIOGRAPHIES}

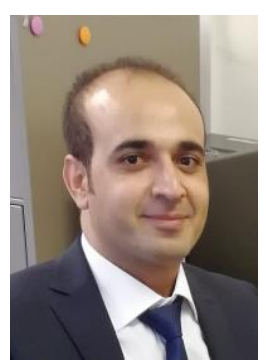

DAVUT IZCI He received his BSc degree from Dicle University, Turkey, in Electrical and Electronic Engineering and his MSc and PhD degrees from Newcastle University, England, UK, in Mechatronics and Microsystems, respectively. $\mathrm{He}$ is currently a member of academic staff at Batman University, Turkey. His research interests are in microsystems development, sensing applications utilizing graphene nanomaterial and robotics along with instrumentation and control systems.

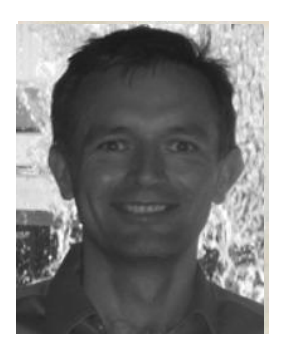

JOHN HEDLEY He received the B.Sc. degree in astronomy and astrophysics and the Ph.D. degree in atomic physics from Newcastle University, U.K., in 1991 and 1996, respectively. After three postdoctoral researcher positions in MEMS design, fabrication, and testing, he obtained an academic position with the School of Engineering, Newcastle University, where he is currently a Senior Lecturer. His research interests are in microsystems development and sensing applications utilizing graphene. 